\title{
Summary of Zhang Hongxing's Experience in Treating Refractory Angina Pectoris with Traditional Chinese Medicine
}

\author{
Fen Han, Hongxing Zhang* \\ Dezhou Hospital of Traditional Chinese Medicine, Dezhou 253000, Shandong Province, China
}

\begin{abstract}
As a famous traditional Chinese medicine (TCM) expert in Shandong province and a famous veteran TCM expert in Dezhou city, Zhang Hongxing has been engaged in cardiovascular clinical work for more than 30 years. He has unique experience in the treatment of refractory angina pectoris. This paper comprehensively explains from the aspects of etiology and pathogenesis. His treatment is mostly based on syndrome differentiation, combined with the basic pharmacology of modern Chinese medicine to formulate prescriptions and drugs, and at the same time values the use of insect drugs. We hereby attach the clinical records, hoping to provide reference for clinical workers, which is conducive to the inheritance and development of famous Chinese medical academic experience.
\end{abstract}

Key words: Zhang Hongxing; Refractory angina pectoris; Traditional Chinese medicine treatment; Famous Chinese medical academic experience

Publication date: May, 2021; Publication online: 31 May, 2021

*Corresponding author: Hongxing Zhang, zhxzhx1967@,163.com

\section{Introduction}

Chief Physician Zhang Hongxing, as a famous traditional Chinese medicine (TCM) expert in Shandong province and a famous TCM expert in Dezhou city, has been engaged in clinical cardiovascular work for more than 30 years. In his long-term of clinical practice, he has accumulated rich experience in the treatment of refractory angina. The author is fortunate to be able to follow him in clinics and has benefited a lot from it. The summary of his experience in the treatment of refractory angina is now summarized, aiming to provide references for passing on academic thoughts of Director Zhang Hongxing as well as experiences to clinical workers in the treatment of refractory angina.

Refractory angina pectoris refers to frequent occurrences of angina pectoris, which cannot be effectively controlled after standard treatment; or recurrent angina pectoris in patients with acute coronary syndrome after percutaneous coronary intervention (PCI) or coronary artery bypass graft $(\mathrm{CABG})$; or angina pectoris caused by the combination of other diseases (such as diabetes, peripheral vascular disease, etc.) not suitable for performing PCI or $\mathrm{CABG}^{[1]}$. With the increase in the number of patients with coronary heart disease (CAD), the number of patients with refractory angina has gradually increased. According to epidemiological survey data, more than 100,000 patients with refractory angina are diagnosed each year ${ }^{[2]}$. The increasing number of patients with refractory angina pectoris poses a major challenge to the current situation of angina pectoris treatment in China, and it also prompts medical workers to continuously explore and try on dealing with it. Director Zhang Hongxing's TCM thoughts and clinical experience in treating this disease are worthy of impartment and development.

\section{Careful examining the etiology and}

\section{pathogenesis}

Refractory angina pectoris belongs to the category of chest pain and heartache in TCM. Both the ancient and modern TCM doctors mostly believe that the main pathological 
factor is deficiency of $q i$ and blood, obstruction of the heart and arteries, the disease is in the heart, and involves the liver, lung, spleen, kidney and other organs. Director Zhang Hongxing recognized that the imbalance between yin and yang, as well as qi and blood, is the root cause of this disease, while phlegm and stasis obstructing the meridians are the symptomatic causes. The deficiency and excess are mixed, and the conditions are complicated. Therefore, the clinical diagnosis should distinguish between clearing $q i$ and yang deficiency, or blood stasis and phlegm turbidity to provide reliable basis for treatment.

The age of onset of this disease is more common in the middle-aged and elderly range. People with weak physique, over 50 years old, halved kidney $q i$, declining vital essence, deficiency in kidney yang, and unable to invigorate the yang of the five internal organs, can cause deficiency of heart $q i$ or heart yang, blood vessels losing warmth, impaired arthralgia, resulting in $q i$ deficiency and blood stasis, when coupled with emotional disorders and eating disorder, can result in poor $q i$ circulation, spleen failure, dampness and phlegm, heart failure, and phlegm production. Director Zhang believes that deficiency of heart qi and heart yang are the root causes of the disease. Insufficient heart $q i$, poor $q i$ circulation, inability of $q i$ to promote blood circulation, and insufficient heart yang to warm the blood veins will result in blood stasis, leading to clinical symptoms such as chest pain, and chest tightness, etc. Blood stasis and phlegm turbidity are pathological products developed on the basis of $q i$ deficiency and yang deficiency, which further aggravate the clinical manifestations. As far as root and symptomatic causes are concerned, the changes in pathology of deficiency in yang qi, blood stasis and phlegm turbidity run through the whole process of coronary heart disease. It is necessary to distinguish between root causes and symtomatic causes, deficiency and excess, light and severe, and between chronic and urgent, and avoid blindly prescribing tonics.

Therefore, it is called refractory angina pectoris because it has a long course, complicated conditions, the ever-changing, lingering and difficult healing, and it is difficult to treat it from a single aspect. Director Zhang pointed out that the pathogenesis of this disease can be summarized as follows: (1) Qi deficiency and blood stasis. Elderly with weak physique, deficiency in normal $q i$, insufficient $q i$ to promote blood circulation, and blood pulse stagnation; clinical patients show chest pain, chest tightness, general fatigue and sweating that aggravate with movement, dark red tongue, white greasy tongue coating, tight or astringent pulse. (2) yang deficiency. This disease "is located in the heart, but its root is in the kidneys." The deficient kidney yang cannot assist the heart, resulting in deficiency of heart yang, which can manifest as faintly pain at the heart, cold limbs, dizziness and tinnitus. (3) Phlegm fire disturbs the heart. $Q i$ stagnation and turbid blood stasis produces phlegm, and phlegm produces heat and fire, drying yin fluid. Chest tightness is the most important clinical manifestation, unbearable pain; palpitation is significant after activity, thirst, red urine, dry and red tongue, deficiency in saliva, and less pulse count. Therefore, the patterns of pathogenesis should be closely followed during treatment, and be flexible to relieve chronic diseases.

\section{About syndrome differentiation and}

\section{treatment}

Director Zhang pointed out that syndrome differentiation needs to be distinguished between deficiency and excess, and between root causes and symptomatic causes. The pathological key of this disease is the deficiency in root causes and the excess in symptomatic causes. The two methods of strengthening the body and dispelling the 'evil' are very important in the treatment. This disease is mostly observed in long-ailing and frail patients, and the clinical symptoms are mostly chest pain and chest tightness. For the main symptoms, the treatment is mostly based on the principle of "dredging by supplement", that is, by supplementing the organs to dredge veins and meridians for better circulation, where the supplementing should focuses on the yang qi, and the dredging should focus on the phlegm and blood stasis. Supplementing and dredging are used in combination to treat phlegm and blood stasis simultaneously, and taking care of both the symptoms and the root causes. Commonly used Chinese medicines include astragalus, Dang Shen (Codonopsis root), dwarf lilyturf tuber, Dong Quai, Chuanxiong (Szechuan lovage root), red peony root, Chinese Red Sage, peach kernel, safflower, corydalis, Gua Lou (Trichosanthes fruit), cinnamon twigs, Chinese aconite, Siberia landpick, epimedium, jujube kernel, Chinese thuja seeds, panax notoginseng powder etc. Director Zhang uses medicines that are warm but not dry, tonic but not stagnant, nourishing but not greasy, to reconcile $q i$ and blood, dredge blood vessels, restore visceral function, remove the cause of disease, and relieve pain. The treatment is mostly based on syndrome differentiation combined with modern TCM 
pharmacology and prescriptions. Insect drugs will be prescribed for patients with more serious illnesses, which have better clinical effects.

\subsection{Identifying main evidence, starting from chest pain and chest tightness}

Identifying the pathogenesis of the main symptoms, assigning prescriptions and medicines suitable for the symptoms, and alleviating long-term refractory symptoms are particularly critical.

\subsubsection{Intractable chest pain}

Mainly due to deficiency of $q i$ and $q i$ stagnation leading to blood stasis, which is painful, and in more severe cases it can be as painful as being pricked by needles. Site of pain is fixed, or radiating to the upper abdomen, back or shoulders, etc. It may be accompanied by chest tightness, palpitation, dizziness, fatigue, cold limbs, dark red tongue, white greasy tongue coating, and tight or astringent pulse. The therapeutic drugs prescribed are mainly those that activate blood circulation, remove blood stasis, and warm and dredge veins. For blood activating and blood stasis removing drugs: Director Zhang uses self-made modified Shenxiong Huoxue Decoction or Xuefu Zhuyu Decoction, and highly values the flexible use of blood activating and blood stasis removing drugs, choosing drugs based on the following four aspects.

\subsubsection{Promoting blood circulation and removing blood stasis}

The patient has recurrent symptoms, extreme pain, and only mild ischemic changes on the ECG, indicating that the symptoms of blood stasis are mild. Blood circulation and nourishing methods are often used, which has mild effects. Use dong quai, Chuanxiong, red peony root, and Chinese Red Sage, etc.; if the angina pectoris is frequent, the pain is severe, and the ECG has obvious ischemic changes, it indicates that the blood stasis is more severe, and treatment method with strong effects is used. Clinically it is used for patients with refractory angina who react poorly to blood nourishing treatment method or have heart failure, as the symptoms cannot be conquered unless this method is used. Most medicines are used to promote blood circulation and remove blood stasis, using peach kernels, safflower, turmeric, rhubarb, leeches, and earthworms, etc.

\subsubsection{Activating blood circulation and relieving pain}

For those with obvious pain, use corydalis, wild ginger, Typha orientalis, boninrousette (Wulingzhi), frankincense, and myrrh, etc.

\subsubsection{Invigorating $q i$ and activating blood}

Deficiency of $q i$ is mainly manifested in the deficiency of vitality and lack of $q i$, as "qi is the commander of blood," $q i$ deficiency results in disability to warm and nourish the blood vessels and promote the normal operation of blood in the vessels, causing blood stasis to block the blood vessels. Use ginseng, Dang Shen, astragalus, and Siberia landpick etc. "Compendium of Materia Medica" written that ginseng has the effects of nourishing $q i$ and calming the mind, raising intelligence and nourishing the mind, and dredging blood veins. Astragalus can replenish all deficiency and is also an essential medicine for $q i$. When used in combination with Shenqi, the effect of replenishing $q i$ is particularly strong. In clinical practice, Dang Shen is often used instead of ginseng, which has a gentle effect on $q i$.

\subsubsection{Regulating $q i$ and activating Blood}

"Qi movement leads blood movement, and qi stagnation causes blood stasis". Qi stagnation and blood stasis are often causal and simultaneous. Director Zhang pays special attention to the dredging of liver qi. Ming Dynasty scholar Xue Ji pointed out in "Medical Cases of Xue Family": "The heart- $q i$ is harmonious if the liver-qi is flowing, and the heart- $q i$ is weak when the liver- $q i$ is stagnant. The liver is the organ for blood storage. It can regulate blood volume, blood flow and the dilation and contraction of meridians. If due to poor emotions, liver loses its functions of dredging and drainage, meridians experience disorders in relaxation and contraction, and heart convulses rapidly, can lead to chest and heart pain. Clinically, it is also common for patients with frustrations. Trifoliate orange, bupleurum, turmeric, Szechwan Chinaberry fruit, and radix lindera etc. are often used to dredge the liver and relieve pain. In terms of warming and tonifying medicine: It is mostly considered from the perspective of warming yang and tonifying the kidney. In the past, some doctors believed that warming medicine increases myocardial oxygen consumption and is not commonly used. Director Zhang believes that "blood flows when it's warm". Warming medicine can make the blood flow unobstructed and improve the symptoms of angina.

\subsection{Warming-yang medicines}

The medicines used are mostly Chinese aconite, cinnamon, cassia twig, wild ginger and other pungent ingredients to warm the interior and dispel cold.

\subsection{Kidney-tonifying drugs}


Medicines, such as cuscuta seed, mulberry mistletoe, Siberia landpick and epimedium are commonly used. Cuscuta seeds can invigorate the kidneys and the liver, warm the spleen and assist the stomach, it is nourishing but not strong, warm but not dry. Upon entering the kidney meridian, it can supplement when there is deficiency, reduce when there is excess, warm-up when there is cold, chill when there is heat, dry-up when it's damp, and moisturize when it's dry. Epimedium enters the two meridians of liver and kidneys, and has the functions of nourishing the kidney and yang, replenishing $q i$ and strengthening the heart.

\subsubsection{Chest tightness}

Long-term illness, coupled with improper diet, loss of health and functions of the spleen, no power to transport and operate, failure of lifting and lowering, inability to transform body fluid, stasis accumulates and transform into phlegm, and turbid phlegm rise up to hurt the chest, blocking chest yang and developing into heart disease, its clinical symptoms are mostly chest tightness and discomfort. Professor Lin treats it from two aspects: cold and heat. For those with cold phlegm, Gualou Xiebai Banxia Decoction or Gualou Xiebai Guizhi Decoction are the main choices; for those with hot phlegm, Xiaoxianxiong Decoction and Wendan Decoction are the main choices. Common prescribed drugs include Chinese goldthread, Gualou, mandarin orange peel, bamboo shavings, and platycodon, etc. If it is due to poor emotions, liver failure, dysfunction of meridian dilation and contraction, and rapid contraction of the heart and collaterals, it can also cause chest tightness. Clinically, it is not uncommon for patients to suffer from emotional frustrations. Director Zhang prescribes modified Chaihu Shugan Powder for those with chest tightness caused by pathogenesis of $q i$ stagnation. To dredge the liver, regulate qi circulation and relieve pain, the medicines are commonly prescribed, including trifoliate orange, bupleurum, turmeric, Szechuan Chinaberry fruit, and radix lindera, etc.

\subsection{Regarding the pharmacology of TCM}

In the 1980s, the research of TCM pharmacology in cardiovascular aspect was flourishing. Through the research of TCM pharmacology, the scientific principles of TCM treatment were revealed and the overall development of Chinese medicine was promoted. Director Zhang highly values Chinese medicines that have been confirmed by clinical pharmacological experiments to strengthen the heart and prevent myocardial ischemia, and use them to improve clinical efficacy. Refractory angina pectoris is mostly caused by hemodynamic disorders due to coronary atherosclerosis, coronary artery spasm, and stent thrombosis, etc ${ }^{[3,4]}$. Chinese Red Sage, Chuanxiong, red peony root, corydalis, safflower, Jixueteng (caulis spatholobi) and other blood-activating and stasis-removing drugs have the effects of dilating coronary arteries, reducing peripheral resistance, improving microcirculation, and increasing myocardial blood and oxygen supply. Ginseng, astragalus, Dang Shen, dwarf lilyturf tuber and other $q i$ and $y i n$ medicines can enhance myocardial contractility, increase cardiac output, increase coronary blood flow, slow down heart rate, and reduce the effects of cardiac preload and afterload. Chinese aconite, cinnamon and other warm medicines have the effects of expanding blood vessels, promoting blood circulation, increasing coronary blood flow, and reducing vascular resistance. Epimedium, cuscuta seed, mulberry mistletoe and other drugs for invigorating kidneys and yang can increase coronary blood flow and improve myocardial cell metabolism. Trifoliate orange can increase coronary blood flow and has diuretic effects. Siler root can relieve pain, and is sedative and anti-allergic. Based on many years of clinical experience, Director Zhang flexibly selects drugs with different efficacy based on the TCM symptoms differentiation combined with the pharmacological characteristics of modern Chinese medicine, which has significant effects on the treatment of patients with refractory angina pectoris.

\subsection{Valuing the use of insect drugs}

In the Qing Dynasty, Ye Tianshi was the first to clearly put forward the theory of "long-term sickness seeps into the meridians" and "long-term pain seeps in the meridians"[5]. Refractory angina pectoris is difficult to heal, conventional drug is difficult to work. Director Zhang believes that the disease has reached the meridians at this stage, and the meridians need to be dredged in order for treatment to be effective. Most insect drugs can reach the meridians and relieve pain, and have a good therapeutic effect for patients with more severe refractory angina pectoris. These medicines can selected, including centipede, stiff silkworm, scorpion, leech, and earthworm, etc. According to the literature, coronary angiography of patients with refractory angina pectoris showed stenosis or blockage of multiple vessels. They are in the state of severe myocardial ischemia and show clinical manifestations of repeated chest pain and 
chest tightness. Insect drugs can get through the veins, relieve meridian stasis, and play an important role in establishing collateral circulation.

\section{Clinical example}

Gao, male, 73 years old, has repeated chest tightness and chest pain for more than 20 years. Over 20 years ago, he had been having chest tightness and chest pain since he go into a situation acting angry, and was diagnosed as "coronary heart disease, unstable angina pectoris" at Dezhou Traditional Chinese Medicine Hospital. After he got better following the PCI surgery (two anterior descending artery stent implantation), he was discharged and advised to take aspirin, Betaloc, Xinkang, atorvastatin, Compound Danshen Dripping Pills, etc. But the chest tightness and chest pain still recurred. The patient had a history of type 2 diabetes, arrhythmia (ventricular premature beats), and lacunar infarction. Electrocardiogram during remission of angina pectoris: Tv1-v5 was low. During the onset of angina pectoris: Tvl-v5 two-way, ST segment vl-v6 level down > $0.1 \mathrm{mv}$. Cardiac color Doppler ultrasound showed that the diastolic function of the left ventricle was reduced, EF64\%, and there was no abnormality in the structure of the heart atrioventricular. The patient went to the office of Director Zhang Hongxing to seek treatment. Symptoms observed: chest tightness, palpitation, chest pain from time to time, needle prick-like pain, aggravated anger after fatigue, weakness of the lower limbs, dreams a lot during sleep, low urine volume, dry stool, dark red tongue, yellow tongue coating, weak pulse string. Director Zhang Hongxing analyzed and pointed out that the patient likely belonged to the category of refractory angina pectoris, combined with pulse diagnosis, syndromes were determined to be $q i$ deficiency and blood stasis, and cardiac blood stasis. The modified Shenxiong Huoxue Decoction was prescribed and the specific dosage was: Astragalus 30g, Dang Shen $15 \mathrm{~g}$, dwarf lilyturf $30 \mathrm{~g}$, five-flavor berry $6 \mathrm{~g}$, dong quai $15 \mathrm{~g}$, red peony $15 \mathrm{~g}$, Salvia root $30 \mathrm{~g}$, corydalis $30 \mathrm{~g}$, Shengdi $15 \mathrm{~g}$, Chinese goldthread $6 \mathrm{~g}$, anemarrhena $10 \mathrm{~g}$, jujube seed $30 \mathrm{~g}$, pinellia 9g, tangerine Peel $6 \mathrm{~g}$, panax notoginseng powder $3 \mathrm{~g}^{\text {ORAL }}$, Chuanxiong $15 \mathrm{~g}$, peach kernel $9 \mathrm{~g}$, safflower $15 \mathrm{~g}$, earthworm 9g, and Cyathula root 15g. After taking 7 doses of the prescription, the symptoms of chest pain disappeared, chest tightness and sleep quality also improved. During the follow-up visit, Director Zhang removed Salvia root, corydalis, and anemarrhena from the prescription. After taking 7 doses of the modified prescription, the symptoms of chest tightness improved significantly, and the stool was normal. On the patient's third visit, considering that the patient is an elderly male with loss of kidney yang, therefore should strengthen the kidney and yang and warm the cold, so the prescription was modified as follows: Astragalus $30 \mathrm{~g}$, Chuanxiong $24 \mathrm{~g}$, ligustrum seed $15 \mathrm{~g}$, gastrodia root $12 \mathrm{~g}$, cinnamon 9g, Astragalus complanatus 30g, Japanese pepper $30 \mathrm{~g}$, Chinese thuja kernel $15 \mathrm{~g}$, jujube kernel $30 \mathrm{~g}$, Chinese goldthread $9 \mathrm{~g}$, anemarrhena $15 \mathrm{~g}$, panax notoginseng powder $3 \mathrm{~g}^{\text {ORAL }}$. It has been more than half a year, and the patient has informed that angina pectoris rarely occurs. Even if it happened occasionally, the symptoms were very mild, the weakness of both lower limbs was improved, the sleep quality was good, and the bowel movement was normal. After that, the ECG chart was checked several times: only Tv1-v5 was low and there was no other ischemic manifestation.

\section{References}

[1] Abbate A, Biondf Z, Occai GG, et al. Recurrent angina after coronary revascularization: A Clinical challenge[J]. Eur Heart J,2007,28(9):1057-1065.

[2] Mukherjee D, Bhatt D, Roe MT, et al. Direct myocardial revascularization and angio-genesis: how many patients might be eligible?[J]. Am J Cardiol,1999,84:598-600.

[3] Filippo O, Nicola B, Casagrandal, et al. Chest pain evaluation project[J]. G ltal Cardiol (Rome),2009,10(1):46-63.

[4] Son V, Phuong CT, Phuong MT, et al. Antithrombotic strategies in patients undergoing percutaneous coronary intervention for acute coronary syndrome $[\mathrm{J}]$. Drug des DevelTher,2010(4):203-220.dol:10.2147/DDDT.s/2056

[5] Dai GH. Lin Huijuan's experience in treating coronary heart disease $[\mathrm{J}]$. Journal of Traditional Chinese Medicine, 2003,44(11):821-822. 